Review

\title{
Nanotechnology, Inflammation and the Skin Barrier: Innovative Approaches for Skin Health and Cosmesis
}

\author{
Angelo Landriscina ${ }^{1}$, Jamie Rosen ${ }^{2}$ and Adam J. Friedman 1,2,* \\ 1 Division of Dermatology, Department of Medicine, Albert Einstein College of Medicine, Bronx, \\ NY 10467, USA; E-Mail: angelo.landriscina@med.einstein.yu.edu \\ 2 Department of Physiology and Biophysics, Albert Einstein College of Medicine, Bronx, \\ NY 10461, USA; E-Mail: jrosen12@gmail.com \\ * Author to whom correspondence should be addressed; E-Mail: adfriedm@montefiore.org; \\ Tel.: +1-718-920-2680.
}

Academic Editor: Lisa A. DeLouise

Received: 3 April 2015 / Accepted: 29 May 2015 / Published: 3 June 2015

\begin{abstract}
Dermatologic physiology and aesthetics are profoundly connected. Inflammatory stimuli abound in the environment, and have the potential to impact both the physiology and aesthetics of the integument. Inflammation results in a compromised epidermal barrier, impaired moisture retention, erythema, scale and pigment alteration. The advent of nanotechnology has introduced a variety of new approaches to preserving skin cosmesis in the face of inflammation. In this article, we review the architecture and physiology of the epidermal barrier, describe the pathological and aesthetic effects of inflammation, and report recent advances in the development of nanomaterials to offset the aesthetic impact of inflammation.
\end{abstract}

Keywords: inflammation; nanotechnology; cosmetics; epidermal barrier; erythema; scale; pigment alteration

\section{Introduction}

The integument serves as the primary interface between the body and its environment. Its position as the most superficial organ renders the skin crucial for interactions with the outside world - be they physical (sensation, defense, regulation) or psychological (interpersonal, intrapersonal, societal). 
The link between these two types of functioning is strong, given the fact that cutaneous pathology is often accompanied by observable changes to the appearance of skin. In this way, physiology and aesthetics are profoundly intertwined.

Inflammation is a primary feature of many dermatologic disease processes. Inflammation has many effects on cutaneous physiology, with the skin's barrier function being especially impaired in inflammatory diseases [1,2]. This disruption can serve as a portal of entry for microbes, allergens and other pro-inflammatory stimuli, which can cause further inflammation. While many of these disease processes are not fully understood, therapies that modulate inflammation have been shown to significantly decrease morbidity due to inflammatory disease. However, inflammation also impacts the appearance of skin in the absence of true pathological processes. Inflammatory stimuli are ubiquitous in the environment, from the microbes that colonize the body, to UV rays and even commonly ingested foods [3]. These factors can result in cutaneous inflammation with varying degrees of physiological and aesthetic impact.

Compounds that prevent and mitigate inflammation and its effects have been an area of increasing interest in the field of cosmetics for some time. The advent of nanotechnology-the use of materials between 1 and $100 \mathrm{~nm}$ in diameter-has provided a new avenue for the delivery of these anti-inflammatory compounds. The use of nanomaterials has allowed for the development of treatments tailored for specific interactions with the skin. These compounds allow for several methods of altering inflammation and its effects on cutaneous physiology, barrier function and aesthetics.

\section{The Skin Barrier-Architecture, Function and the Impact of Inflammation}

The primary agent of the skin's barrier function is its outermost layer, the stratum corneum. The stratum corneum is composed of corneocytes (anucleated squamous cells with a high content of keratin filaments and an envelope composed mainly of cross-linked proteins and covalently bonded lipids) linked together by corneodesmosomes and nonpolar extracellular lipids (Figure 1) [4]. These components are the product of a long maturation process that starts in the basal layer. Keratinocytes undergo several structural and compositional changes as they ascend to the stratum corneum. As the keratinocytes mature, they also expel lamellar bodies-organelles packed with glycosphingolipids, free sterols and phospholipids, which serve as the precursors to the lipid portion of the stratum corneum (composed primarily of ceramides, cholesterol and free fatty acids) [4].

The tight network of corneocytes and lipids in the stratum corneum makes percutaneous absorption of substances difficult, though some key principles can be exploited in order to traverse the epidermal barrier. Diffusion across the stratum corneum is dictated by the size, lipophilicity and charge of the molecule in question [4]. Furthermore, there are three possible routes of absorption: transcellular (through corneocytes), intercellular (through the extracellular space), and transappendageal (through accessory structures such as follicular openings). Topical applications can alter permeability through moisturization, depolarization of the substance to be absorbed, and through the use of detergents and other permeability-enhancing agents [4]. 


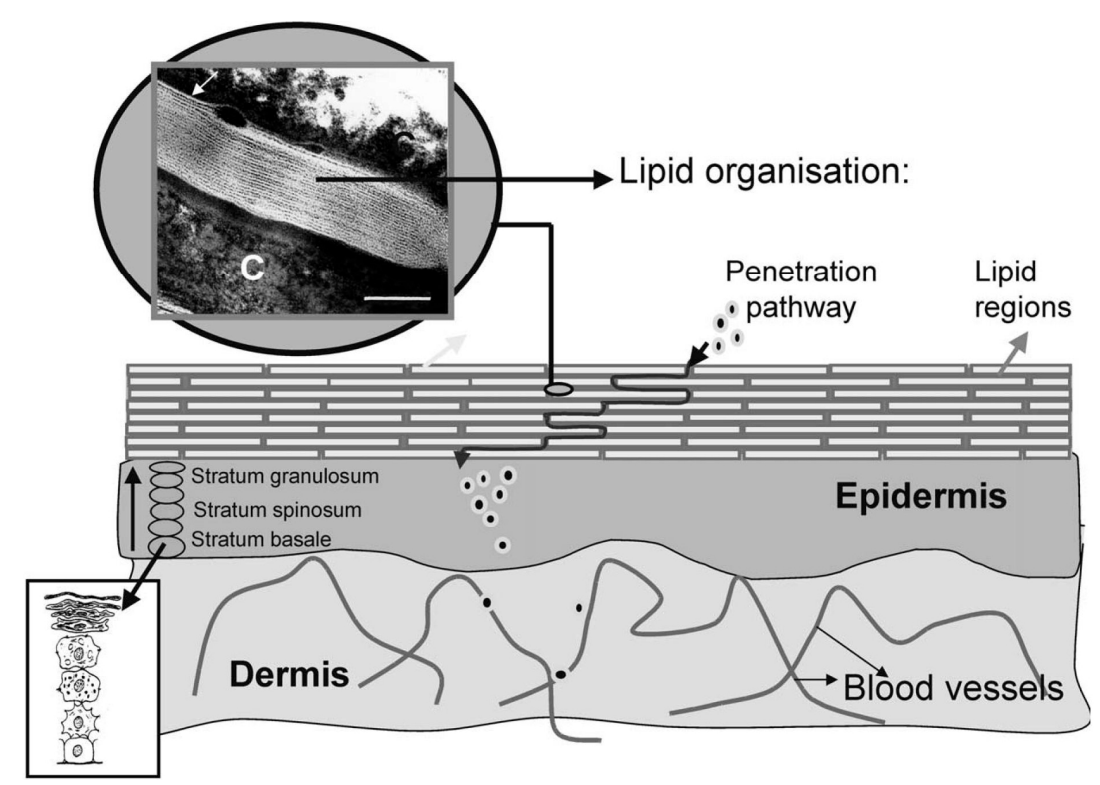

Figure 1. Architecture of the Epidermal Barrier. This schematic of the skin in cross section illustrates the architecture of the epidermal barrier. Keratinocyte maturation occurs as cells progress to the stratum corneum, at which time they differentiate into corneocytes. The corneocytes are surrounded by extracellular lipids and cross-linked proteins. $\mathrm{C}=\mathrm{a}$ micrograph showing a corneocyte filled with keratin. Scale bar $=100 \mathrm{~nm}$. Adapted with permission from Elsevier, 2003 [5].

The study of inflammatory disease has underscored the effects of inflammation on the epidermal barrier. For instance, the skin of patients with atopic dermatitis, a chronic inflammatory disease of the skin with a $\mathrm{T}_{\mathrm{H}} 2$-predominant immune response, exhibits a deficiency of ceramides, corneodesmosomes, and filaggrin - a protein important for the cross-linking of keratin fibers $[2,6]$. The deficiency of these factors has been specifically linked to increased production of several pro-inflammatory cytokines [1,2]. The result of these changes is an impairment of barrier function, leaving the skin more susceptible to water loss and entry of allergens and other pro-inflammatory molecules, creating a vicious cycle of inflammation and further defortification of the epidermal barrier. While patients without inflammatory dermatological conditions are less susceptible to these types of insults, the principles still hold true, with inflammatory substances abounding in the environment [3].

\section{The Aesthetic Impact of Inflammation}

Inflammation and the resultant weakening of the epidermal barrier have several implications for cutaneous aesthetics. The most immediate effect is erythema. Inflammatory cells release a variety of vasoactive substances including nitric oxide (NO) and vascular endothelial growth factor [7]. The release of these substances results in vasodilation, intended to increase further recruitment of inflammatory cells. The aesthetic result of this process is erythema-the appearance of a reddish hue on the skin (Figure 2A). The second visual effect of inflammation is scale (Figure 2B). As mentioned above, inflammation results in the down regulation of many of the molecules that form a cohesive stratum corneum including filaggrin and corneodesmosomes. As a result, collections of corneocytes can disadhere from the stratum corneum. The result is a white flakiness, commonly referred to as scale. 
Moisture loss due to impaired barrier function, and hyperproliferation of the epidermis (common in inflammatory conditions), can also fuel this process. A third aesthetic effect of inflammation is pigment alteration. Post-inflammatory pigment alteration (PIPA) is commonly seen in resolving inflammatory lesions (Figure 2C). The effect is most often hyperpigmentation in the area of the resolving lesion, and is thought to be the result of the reaction of melanocytes to pro-inflammatory cytokines [8]. PIPA can last for months after inflammation has resolved, and more commonly affects patients with darker Fitzpatrick skin types [8]. These three effects together have a considerable impact on cutaneous aesthetics.
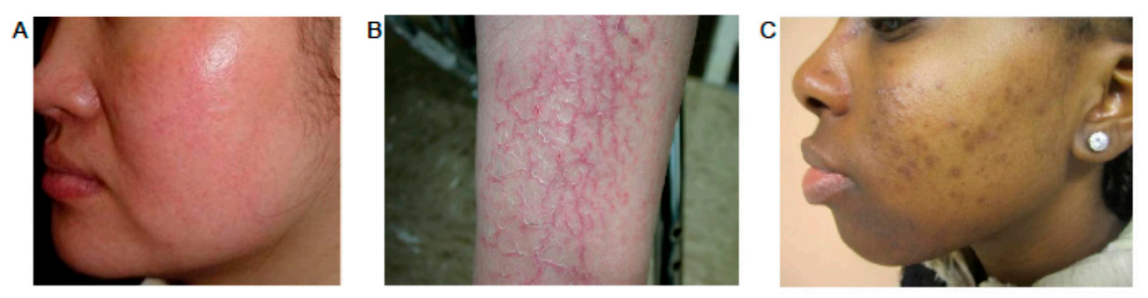

Figure 2. The aesthetic impact of inflammation. (A) A patient with persistent facial erythema due to rosacea. (B) Scale in a patient with atopic dermatitis. (C) Post-inflammatory pigment alteration in a patient with acne vulgaris. Adapted with permission from Elsevier, 2012 [9]; with patient permission from Alexis, A; with permission from Springer, 2014 [8], respectively.

\section{Nanomaterials-A Primer}

While there has been much controversy about the definition of "nanotechnology," it is generally accepted that nanomaterials are those with a particulate diameter of 1-100 nm. While nanomaterials have been used by humans for thousands of years [10], the last decade has seen a tremendous growth in the use of nanomaterials in consumer products, with greater than $13 \%$ of these products being classified as cosmetics [4]. The rapid growth of nanomaterial development has caused some concern among consumers, with $64 \%$ claiming that they would not use cosmetics that contained nanomaterials [4]. Interestingly, many consumers are unsure of exactly what constitutes nanotechnology [4].

Nanoscale size imparts a variety of desirable properties, especially when considering their use in topical applications. Firstly, their small size allows for penetration past most physiological barriers - an especially advantageous property when considering the epidermal barrier. The small size of nanoparticles also allows for a higher surface area to volume ratio, allowing for greater exposure of active molecules per dose administered [11]. Nanoencapuslation also allows for sustained release, and provides a new method for delivering unstable or insoluble compounds [12]. These properties of nanomaterials are also modifiable - particle size can be altered to control penetration, pore size to control release, surface molecules added for targeting to specific tissues - allowing nanotherapies to be tailored for distinct uses.

Size and surface charge are especially important when considering transdermal permeation of nanomaterials. Passive transport of nanomaterials through the epidermal barrier is unlikely due to the factors mentioned previously, as well as the protein-rich layers below the stratum corneum [4]. Passage via the transcellular route is highly dependent on charge and lipophillicity, with nonpolar 
substances more likely to enter cells, and decreases in size resulting an in exponential increase in permeability [13]. Penetration through the intercellular route is also limited, with impermeability to particles greater than $36 \mathrm{~nm}$ [14]. The transappendageal route, in contrast, may provide a significant point of entry for nanomaterials, with permeability to particles less than $200 \mu \mathrm{m}$ [14]. Based on these principles, the majority of nanomaterials should not be systemically absorbed, with some evidence indicating that only particles less than $10 \mathrm{~nm}$ exhibit transdermal absorption [15]. However, the impairment of epidermal barrier function seen in inflammatory disease may allow for greater absorption of nanomaterials. This important difference should inspire caution in the development of nanotherapies, but also provide an opportunity for the development of nanomaterials that selectively penetrate inflamed skin.

\section{Nanotherapeutics as a Treatment for Inflammation}

\subsection{Prevention and Direct Inhibition of Inflammation}

The most direct approach for correcting the aesthetic effects of inflammation is to inhibit the inflammatory cascade. Many mainstays of dermatologic therapy aim to prevent or attenuate inflammation. For instance, topical corticosteroids are a cornerstone of dermatologic therapy, and have been used for a wide variety of conditions since their introduction in the 1950s. They are used both for the treatment and the prevention of cutaneous inflammation [16]. They are often prescribed for minor inflammatory events, though their use can carry significant side effects, with cutaneous atrophy being one of the most common. This phenomenon is due to thinning of the skin, resulting from the inhibition of fibroblasts and collagen cross-linking by corticosteroids. Adverse effects like these make a glucocorticoid formulation with predictable penetration and concentration desirable. Santos Maia et al. found that a solid lipid nanoparticle (SLN) corticosteroid formulation showed a localizing effect to the epidermis in vitro, which they posit could prevent atrophy [17]. However, several studies have shown increased penetration of other SLN glucocorticoid formulations compared to its traditional non-nano counterparts, resulting in penetration beyond the epidermis and into the dermis $[16,18]$. Nevertheless, SLN technology provides the added benefit of sustained release, a desirable property for controlling concentration of drug in tissue over time, another feature that may improve side effect profiles and dosing schedules, though further study is needed to fully evaluate this technology [16].

Sunscreens are an invaluable tool for skincare and a first line agent for the prevention of inflammation caused by ultraviolet (UV) rays. UV filters are integral for the prevention of sunburn, photo-aging and UV-induced mutations that may lead to malignancy. The inorganic UV filters, titanium oxide $\left(\mathrm{TiO}_{2}\right)$ and zinc oxide $(\mathrm{ZnO})$, have been used in sunscreen formulations for years and are effective in protecting against both UVA and UVB rays. However, use of these filters has been limited due to a lack of cosmetic elegance. The raw materials of these inorganic filters are coarse white powders that reflect light in the visible spectrum. As a result, sunscreens using $\mathrm{TiO}_{2}$ and $\mathrm{ZnO}$ leave an opaque white residue on the skin, a factor that leads many consumers to choose other UV filters. By decreasing $\mathrm{TiO}_{2}$ and $\mathrm{ZnO}$ aggregate size to the nanoscale, the production of translucent $\mathrm{TiO}_{2}$ and $\mathrm{ZnO}$ sunscreens is possible (Figure 3). It has been shown that sunscreens using particles between 40 and $60 \mathrm{~nm}$ are capable of providing good transparency without compromising UVA and UVB 
attenuation [19]. While there has been some concern about the use of nanomaterials in sunscreens, especially when utilizing inorganic compounds, there is no indication that use of $\mathrm{TiO}_{2}$ or $\mathrm{ZnO}$ poses any danger to consumers via systemic absorption, with studies showing that nanoparticle preparations do not penetrate past the stratum corneum [20]. Further study is needed to fully evaluate the safety of these filters.

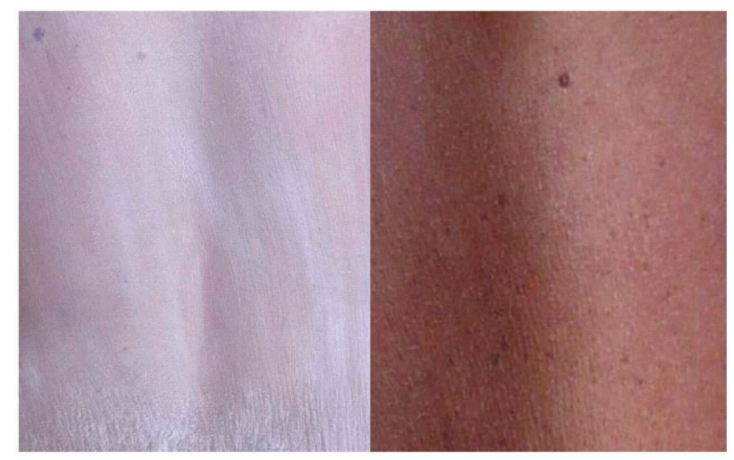

Figure 3. Side by side comparison of traditional and nanosized zinc oxide sunscreen. Photos showing appearance of the skin after six applications of non-nanosized (left) and nanosized (right) $\mathrm{ZnO}$ sunscreens. Adapted with permission from Oxford University Press, 2010 [21].

\subsection{Moisturization and Fortification of the Skin Barrier}

Fortification of the skin barrier is desirable in the setting of inflammation. As mentioned above, when the epidermal barrier is weakened by inflammatory activity, the skin's ability to regulate water loss and prevent the entry of foreign substances is compromised. The aesthetic outcome of these changes is the appearance of scale, and further stimulation of inflammation. Classically, the use of barrier creams, thick water in oil or oil in water emulsions, has been thought to improve barrier function in inflammatory conditions by providing occlusion and trapping or transforming allergens and other irritant molecules [22]. However, patient compliance to the use of these preparations is impacted by their cosmetic properties - highly occlusive preparations tend to have a noticeable shiny or partially opaque appearance and greasy texture. Nanomaterials have been incorporated into new barrier cream formulations in order to provide increased cosmetic appeal, more elegant textures and added benefits. These nanoparticulate formulations are superior to traditional creams in their ability to minimize water loss and prevent irritation while producing formulations with a thinner texture [23]. Wissing and Muller found that oil in water emulsion incorporating SLN provided a significantly improved hydration benefit over a conventional moisturizer in a human trial [24]. Furthermore, the SLN platform is customizable and can be tailored to provide varying levels of occlusive effect for specific skin conditions [25]. Moisturizers incorporating nanomaterials have also been found to provide additional cosmetic benefits. For instance, Kato et al. demonstrated that a nanoprepartion of fullerene dissolved in squalene not only minimized water loss, but also resulted in significant improvement in the appearance of wrinkles [26]. These nanoplatforms show promise for combating scale associated with inflammation, though larger scale trials are needed to fully evaluate efficacy and safety. 


\subsection{Color Correction}

As mentioned above, two of the primary aesthetic effects of inflammation result in alteration of color: erythema and PIPA. In order to conceal these changes, many consumers turn to the use of traditional makeup (foundations, concealers) in order to temporarily color correct their skin. Nanomaterials, especially metal-based nanopigments, have garnered interest for use in color cosmetics due to their optical properties. For instance, Alfano et al. proposed a method of color correction using a two-color system [27]. The first color resembles the user's normal skin tone and is achieved using primary particles of $\mathrm{ZnO}$, magnesium oxide or titania. The secondary color is meant to modulate unwanted hues in the skin, and employs specific size silver nanospheres in order to provide undertones that will diminish the unwanted color. For instance, a green undertone can minimize the appearance of redness due to erythema, or blue and yellow hues to provide a lightened appearance in the setting of hyperpigmentation. Ha et al. also described a system using variably sized gold and silver nanomaterials emitting red, yellow and blue colors for uses in various powders, foundations, concealers and color cosmetics [28]. Cassin et al. designed a nanoparticle platform using the rare earth metals scandium, yttrium and lanthanide in order to provide the appearance of lighter skin [29]. These nanoparticles absorb UV light and emit longer wavelengths in order to provide a long-lasting bleaching effect, which may be useful in the setting of hyperpigmentation. Unfortunately, many of these technologies are still in the early stages of development, with no in vivo data about efficacy or safety.

Color correction in the setting of hyperpigmenation can also be achieved via the use of bleaching agents. One such agent is all-trans retinoic acid (atRA), a vitamin A derivative that lightens skins by accelerating epidermal turnover and melanin granule discharge [30]. While atRA is efficacious in treating hyperpigmentation, its poor stability in heat, air and light has limited its use. The encapsulation of atRA in nano structures such as micelles and nanoliposomes has been shown to stabilize atRA, extending the shelf life of atRA-containing preparations [31]. Nano-atRA preparations have also shown similar efficacy to traditional topical preparations with improved permeation into the stratum corneum, an attenuated side effect profile, and the ability for sustained release [32]. Skin whitening efficacy has also been achieved using a variety of bleaching agents encapsulated in chitosan nanoparticles, with promising in vivo results [33].

\section{Conclusion}

Inflammation is a ubiquitous phenomenon with significant impact on cutaneous physiology and aesthetics. Traditional skin care agents have aimed to prevent and attenuate the effects of inflammation, including barrier dysfunction, erythema, scale and pigment alteration, in order to maximize both skin health and cosmesis. Nanotechnology has provided a new avenue for the prevention and treatment of inflammation and its sequelae, with particularly promising advances for the augmentation of aesthetics. Future studies are necessary to evaluate systemic absorption and toxicity in order to ensure consumer safety. 


\section{Author Contributions}

Angelo Landriscina conceived, researched, and produced the manuscript; Jamie Rosen consulted on the production and took part in editing of the manuscript; Adam J. Friedman supervised the conception and production and took part in the editing of the manuscript.

\section{Conflicts of Interest}

The authors declare no conflict of interest.

\section{References}

1. Hvid, M.; Johansen, C.; Deleuran, B.; Kemp, K.; Deleuran, M.; Vestergaard, C. Regulation of caspase 14 expression in keratinocytes by inflammatory cytokines - A possible link between reduced skin barrier function and inflammation? Exp. Dermatol. 2011, 20, 633-636.

2. Hvid, M.; Vestergaard, C.; Kemp, K.; Christensen, G.B.; Deleuran, B.; Deleuran, M. Il-25 in atopic dermatitis: A possible link between inflammation and skin barrier dysfunction\&quest. $J$. Investig. Dermatol. 2011, 131, 150-157.

3. McDade, T.W. Early environments and the ecology of inflammation. Proc. Natl. Acad. Sci. 2012, 109, 17281-17288.

4. Mihranyan, A.; Ferraz, N.; Strømme, M. Current status and future prospects of nanotechnology in cosmetics. Prog. Mater. Sci. 2012, 57, 875-910.

5. Bouwstra, J.A.; Honeywell-Nguyen, P.L.; Gooris, G.S.; Ponec, M. Structure of the skin barrier and its modulation by vesicular formulations. Prog. Lipid Res. 2003, 42, 1-36.

6. Imokawa, G.; Abe, A.; Jin, K.; Higaki, Y.; Kawashima, M.; Hidano, A. Decreased level of ceramides in stratum corneum of atopic dermatitis: An etiologic factor in atopic dry skin? $J$. Investig. Dermatol. 1991, 96, 523-526.

7. Broughton, G.; Janis, J.E.; Attinger, C.E. The basic science of wound healing. Plast. Reconstr. Surg. 2006, 117, 12S-34S.

8. Nijhawan, R.; Alexis, A. Post-inflammatory pigment alteration. In Acneiform Eruptions in Dermatology; Zeichner, J., Ed.; Springer: New York, NY, USA, 2014; pp. 279-287.

9. Hsu, C.-C.; Lee, J.Y.-Y. Pronounced facial flushing and persistent erythema of rosacea effectively treated by carvedilol, a nonselective $\beta$-adrenergic blocker. J. Am. Acad. Dermatol. 2012, 67, 491-493.

10. Haveli, S.D.; Walter, P.; Patriarche, G.; Ayache, J.; Castaing, J.; Van Elslande, E.; Tsoucaris, G.; Wang, P.-A.; Kagan, H.B. Hair fiber as a nanoreactor in controlled synthesis of fluorescent gold nanoparticles. Nano Lett. 2012, 12, 6212-6217.

11. Kim, B.Y.; Rutka, J.T.; Chan, W.C. Nanomedicine. New Engl. J. Med. 2010, 363, 2434-2443.

12. Han, G.; Friedman, A.J.; Friedman, J.M. Nitric oxide releasing nanoparticle synthesis and characterization. In Nitric oxide; Springer: New York, NY, USA, 2011; pp. 187-195.

13. Potts, R.O.; Guy, R.H. Predicting skin permeability. Pharm Res. 1992, 9, 663-669.

14. Baroli, B. Penetration of nanoparticles and nanomaterials in the skin: Fiction or reality? J. Pharm. Sci. 2010, 99, 21-50. 
15. Karadzovska, D.; Brooks, J.D.; Monteiro-Riviere, N.A.; Riviere, J.E. Predicting skin permeability from complex vehicles. Adv. Drug Deliv. Rev. 2013, 65, 265-277.

16. Schlupp, P.; Blaschke, T.; Kramer, K.D.; Holtje, H.D.; Mehnert, W.; Schafer-Korting, M. Drug release and skin penetration from solid lipid nanoparticles and a base cream: A systematic approach from a comparison of three glucocorticoids. Skin Pharmacol. Physiol. 2011, 24, 199-209.

17. Santos Maia, C.; Mehnert, W.; Schaller, M.; Korting, H.C.; Gysler, A.; Haberland, A.; Schafer-Korting, M. Drug targeting by solid lipid nanoparticles for dermal use. J. Drug Target. 2002, 10, 489-495.

18. Maia, C.S.; Mehnert, W.; Schäfer-Korting, M. Solid lipid nanoparticles as drug carriers for topical glucocorticoids. Int. J. Pharm. 2000, 196, 165-167.

19. Wiechers, J.W.; Musee, N. Engineered inorganic nanoparticles and cosmetics: Facts, issues, knowledge gaps and challenges. J. Biomed. Nanotechnol. 2010, 6, 408-431.

20. Filipe, P.; Silva, J.N.; Silva, R.; Cirne de Castro, J.L.; Marques Gomes, M.; Alves, L.C.; Santus, R.; Pinheiro, T. Stratum corneum is an effective barrier to $\mathrm{TiO}_{2}$ and $\mathrm{ZnO}$ nanoparticle percutaneous absorption. Skin Pharmacol. Physiol. 2009, 22, 266-275.

21. Gulson, B.; McCall, M.; Korsch, M.; Gomez, L.; Casey, P.; Oytam, Y.; Taylor, A.; McCulloch, M.; Trotter, J.; Kinsley, L.; et al. Small amounts of zinc from zinc oxide particles in sunscreens applied outdoors are absorbed through human skin. Toxicol. Sci. 2010, 118, 140-149.

22. Zhai, H.; Maibach, H.I. Barrier creams-Skin protectants: Can you protect skin? J. Cosmet. Dermatol. 2002, 1, 20-23.

23. De Fine Olivarius, F.; Hansen, A.B.; Karlsmark, T.; Wulf, H.C. Water protective effect of barrier creams and moisturizing creams: A new in vivo test method. Contact Dermat. 1996, 35, 219-225.

24. Wissing, S.A.; Muller, R.H. The influence of solid lipid nanoparticles on skin hydration and viscoelasticity - in vivo study. Eur. J. Pharm. Biopharm. 2003, 56, 67-72.

25. Wissing, S.; Lippacher, A.; Muller, R. Investigations on the occlusive properties of solid lipid nanoparticles (SLN). J. Cosmet. Sci. 2001, 52, 313-324.

26. Kato, S.; Taira, H.; Aoshima, H.; Saitoh, Y.; Miwa, N. Clinical evaluation of fullerene-C60 dissolved in squalane for anti-wrinkle cosmetics. J. Nanosci. Nanotechnol. 2010, 10, 6769-6774.

27. Alfano, R.; Ni, X.; Zevallos, M. Changing Skin-Color Perception Using Quantum and Optical Principles in Cosmetic Preparations. Patent US 11/656,738, 27 August 2007.

28. Ha, T.H.; Jeong, J.Y.; Jung, B.H.; Kim, J.K.; Lim, Y.T. Cosmetic Pigment Composition Containing Gold or Silver Nano-Particles. Patents US20090022765 A1, 22 January 2009.

29. Cassin, G.; Simonnet, J.T. Cosmetic compositions comprising photoluminescent nanoparticles and at least one rare-earth metal. Patent WO2006054202 A1, 26 May 2006.

30. Yoshimura, K.; Tsukamoto, K.; Okazaki, M.; Virador, V.M.; Lei, T.C.; Suzuki, Y.; Uchida, G.; Kitano, Y.; Harii, K. Effects of all-trans retinoic acid on melanogenesis in pigmented skin equivalents and monolayer culture of melanocytes. J. Dermatol. Sci. 2001, 27, 68-75.

31. Schafer-Korting, M.; Mehnert, W.; Korting, H.C. Lipid nanoparticles for improved topical application of drugs for skin diseases. Adv. Drug Deliv. Rev. 2007, 59, 427-443.

32. Gatti, R.; Gioia, M.G.; Cavrini, V. Analysis and stability study of retinoids in pharmaceuticals by LC with fluorescence detection. J. Pharm. Biomed. Anal. 2000, 23, 147-159. 
33. Morganti, P.; del Ciotto, P.; Carezzi, F.; Guarneri, F.; Yeo, Y.J. Skin lightening efficacy of new formulations enhanced by chitin nanoparticles delivery system. Note I. J. Appl. Cosmetol. 2014, $32,57-71$.

(C) 2015 by the authors; licensee MDPI, Basel, Switzerland. This article is an open access article distributed under the terms and conditions of the Creative Commons Attribution license (http://creativecommons.org/licenses/by/4.0/). 